\title{
Aviation Wiring Networks Fault Modeling and Simulation Based on Reflectometry
}

\author{
Xudong Shi ${ }^{1,2,3}$, Chengzong Liu ${ }^{1}$, Yulei Xu ${ }^{1}$, Zhangang Yang ${ }^{1,2,3}$ \\ ${ }^{1}$ Aeronautical Automation College of Civil Aviation University of China, Tianjin, 300300, China \\ ${ }^{2}$ Tianjin Key Laboratory for Civil Aircraft Airworthiness and Maintenance of Civil Aviation University of China, \\ Tianjin, 300300, China \\ ${ }^{3}$ Ground Support Equipments Research Base of Civil Aviation University of China, Tianjin, 300300, China
}

\begin{abstract}
This paper presents a method to model and simulate the aviation branched wiring networks. According to the characteristics of the wiring networks faults, establishing a mathematical model of the branched wiring networks faults based on the reflectometry. Simulation reveals the propagation of highfrequency signal of reflectometry in the aviation branched wiring networks. Establish the corresponding mathematic model and run the wiring networks fault simulation in MATLAB/Simulink. Then analyze the relationship between the real faulty reflected signal and the simulation faulty reflected signal.
\end{abstract}

Index Terms - reflectometry, branched wiring networks, fault location, simulation and modeling

\section{Introduction}

Airplane wiring system supplies the airplane`s power and the transports the signal for transmission and controlling of the plane, which is composed of connecting wires, connectors and many other devices. Airborne equipment includes power systems, hydraulic systems, fuel systems, air conditioning systems, flight control systems, electrical systems, navigation systems, communication systems, landing gear systems, and various types of display devices. The transmission of Airborne equipments` signals relies on this system, which plays a key role in the flight control and safety[1].

Based on the reflectometry we establish mathematical model of the aircraft branched wiring networks, and run the simulation in MATLAB/Simulink software circumstance. Then analyze the real faulty reflected signal and the simulation faulty reflected signal.

\section{Aviation Wiring Networks Fault and the Mathematic Model}

Normally there are three kinds of faults in the wire, such as open circuit fault, short circuit fault and high resistance fault. Aviation branched wiring networks have its own particularity, in the maintenance, the faults of open-circuit, short-circuit and the insulation fray happen frequently, but rarely the high resistance fault[2].

According to the transmission line theory, sending the test signal to the end of a limited wire and the signal will transport along the wire line, if the wire is normal and the terminal load impedance equals to the characteristic impedance of the wire, the signal will be absorbed by the load without reflecting back. If there is a fault, the impedance of fault point will be different from the characteristic impedance of the wire so the reflected signal will generate. The reflection coefficient is the physical quantity to describe the reflection:

$$
\rho=\frac{U_{\text {reflect }}}{U_{\text {incident }}}=\frac{Z_{L}-Z_{O}}{Z_{L}+Z_{O}}
$$

Where the $\rho$ is the reflection coefficient, $Z_{L}$ and $Z_{O}$ are the impedance of the wire before and after the fault point, $\mathrm{U}_{\text {reflect }}$ is the reflected signal voltage, $\mathrm{U}_{\text {incident }}$ is the incident signal voltage.

From equation (1), it can be inferred that: 1 ) when $\rho=0$, $\mathrm{Z}_{\mathrm{L}}=\mathrm{Z}_{\mathrm{O}}$, it means that the wire is fine without faults. The load impedance of the wire matches the characteristic impedance . There is no reflected signal generated with the test signal absorbed by the load. 2) when $\rho=-1, Z_{L}=0$, it means that the short-circuit fault occurs. The reflected signal fully reflects at the fault point. The amplitude of the reflected signal equals to the amplitude of the incident signal, but with the opposite polarity. 3) when $\rho=1, Z_{L}$ approaches infinity. The opencircuit fault occurs. The signal directly reflects back without reversing the polarity[3].

The theoretical foundation of the equivalent model of the wire is the transmission line theory. It can be shown from Fig. 1: According to the transmission line theory, the wire can be considered as a circuit constituted of a number of equivalent resistance $\mathrm{R}$, conductance $\mathrm{G}$, inductance $\mathrm{L}$ and capacitance $\mathrm{C}$. Each parameter uniformly distributed along the entire wire. When the incident signal passes through the wire, by unit length the resistance $\mathrm{R}$ and inductance $\mathrm{L}$ will be a pressure reduction and the signal will shunt by inductance $\mathrm{L}$ and capacitance $\mathrm{C}$ to return back[4].

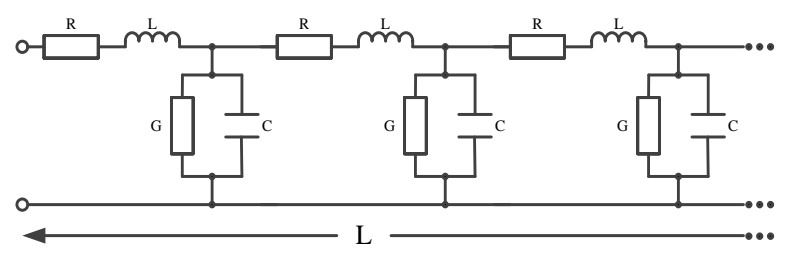

Fig. 1 Equivalent model of the wire

The equation to describe the relationship of the voltage and current at any time and position:

$$
\left\{\begin{array}{l}
-\frac{\partial u}{\partial x}=R i+L \frac{\partial i}{\partial t} \\
-\frac{\partial i}{\partial x}=G i+C \frac{\partial u}{\partial t}
\end{array}\right.
$$


In equation (2): R, L, G, C are physical quantities of unit length; $u, i$ are the voltage and current of distance $x$ from the beginning. When there is a impedance mismatch, the signal will reflect and transport. Assuming that the impedance of the fault section and non-fault section is uniform, the change of the geometric parameters of the fault point will cause the characteristic impedance change. Due to the multi-branches networks, the reflected signal of the branched wiring networks has its own particularity: the impedance mismatch not only happens at the fault point of the wire but also at the transmission branch. And due to the reflection, multireflections occur at the fault point and transmission branch junctions. Also, the signal will attenuate and interfere to bring about the difficulty of fault locating.

When a junction between two wires of equal impedance $Z_{O}$ is encountered, the effective impedance is $Z_{0} / 2$, the coefficient $\rho$ is $1 / 3$, and the transmission coefficient $\tau$ is $2 / 3$. For example, a junction branches into two wires(all wires have the same impedance $Z_{O}$ ), resulting in the impedance of $\mathrm{Z}_{\mathrm{i}}=\mathrm{Z}_{\mathrm{O}} / 2$ and the reflection coefficient of $-1 / 3$. In general, for a junction of $n$ branches of equal impedances, $Z_{i}=Z_{O} / n$, and the reflection coefficient is given by[5]

$$
\rho=\frac{Z_{i}-Z_{o}}{Z_{i}+Z_{o}}=\frac{Z_{o} / n-Z_{o}}{Z_{o} / n+Z_{o}}=\frac{1-n}{1+n}
$$

In Fig 2, it is a further explanation of the course of branched wiring networks multi-reflections and transmission. Thus, the propagation of the reflected signal of a branched wiring networks has been formed, there are a number of equidistant reflected signal after the first reflected signal back from the fault point. Because the signal reflected back and forth between the test point and the junction or fault point. During the propagation there is loss of the signals, and the amplitude of the reflected signal decreases too. Actually the useful reflected signal is the first reflection, the others can not be analyzed as the fault reflected signals[6].
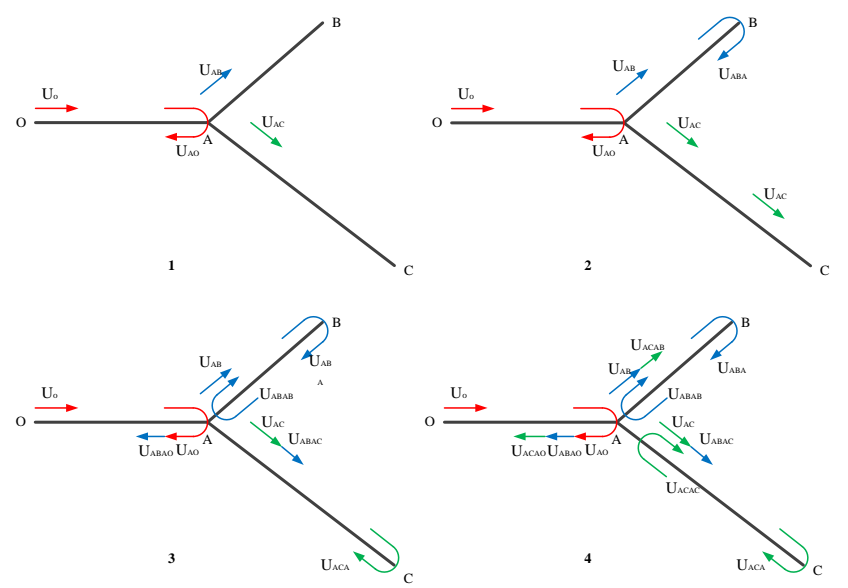

Fig. 2 Illustration of the reflected signal of branched wiring networks

Based on the illustration in Fig 2, the reflected signal propagation of the 2 branched wiring networks can be described in a mathematical model as shown in Fig 3:

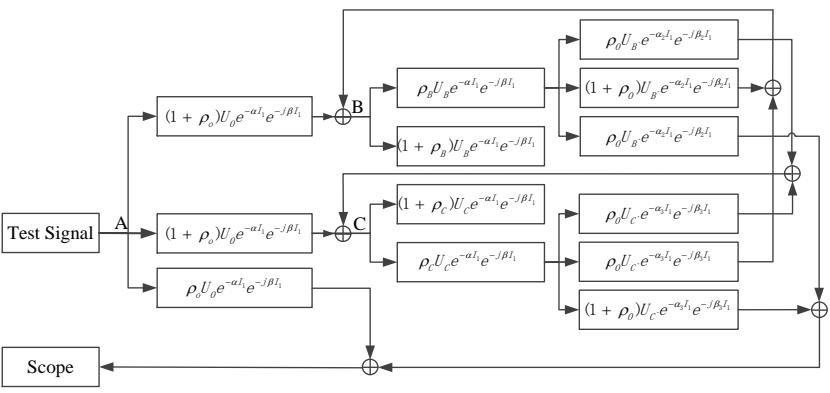

Fig. 3 The mathematical model of the reflected signal of the branched wiring networks

In Fig 3: $\rho_{1}, \rho_{2}, \rho_{3}$ are the reflection coefficient of the branch junction $\mathrm{A}$, the branch end $\mathrm{B}$ and $\mathrm{C} ; \alpha_{1}$ and $\beta_{1}, \alpha_{2}$ and $\beta_{2}, \alpha_{3}$ and $\beta_{3}$ are the attenuation constant and the phase shift constant of $\mathrm{OA}, \mathrm{AB}$ and $\mathrm{BC} ; \mathrm{L}_{1}, \mathrm{~L}_{2}, \mathrm{~L}_{3}$ are the lengths of $\mathrm{OA}$, $\mathrm{AB}, \mathrm{AC} ; \mathrm{U}_{0}$ is the voltage amplitude of the initial test signal, the remaining $\mathrm{U}_{\mathrm{AA}}, \mathrm{U}_{\mathrm{AB}} \cdots \cdots \mathrm{U}_{\mathrm{ACAC}}$, respectively are the voltage amplitude of incident or reflected signals of every characteristic impedance changing point(the fault point or the branch junction point) of the wiring networks, the values of which varies with the times of reflections and transmissions. This model can be extended to other structures of the wiring networks and other types of the faults[7].

When detecting the fault location of the branched wiring networks in reflectometry, we select the high-frequency signal as the test signal. Because the resistance of the wire is small and the insulation resistance is high, in fact, when the high frequency signal reaches to a certain level, the effects of the resistance and conductivity can be negligible, so when establishing the simulation model of the branched wiring networks, only considering the effect of capacitance and inductance. The $\rho$ is real and its value range from -1 to +1 . Therefore, the mathematical model in Fig 3 can be simplified to transport delay units, reflection and transmission units. The simulation can be easily implemented in the MATLAB/Simulink.

The simulation model of the branched wiring networks is shown in Fig 4. In each section of which there are the transport delay units, the reflection or transmission units, and the branched wiring networks multiple reflections feedback loops. Firstly, the signal reaches junction A, Then it divided into 3 signals: the reflected signal back to $\mathrm{O}$, the transmission signals to B and C. In the upper part of Fig 4, it shows the propagation of the signal in branch $\mathrm{AB}$. After the transport delay units and reflection units the signal reaches back to junction $\mathrm{A}$ and be divided into 3 directions: the feedback signal to $\mathrm{C}$, the feedback to $\mathrm{O}$ and $\mathrm{B}$. Because it is a shortcircuit fault in the end of branch $\mathrm{AB}$, the reflected signal is the opposite phase with the incident signal and the value of the reflection unit is -1 . It is similar that the propagation of the signal in branch AC. But the fault in the end $\mathrm{C}$ is open-circuit fault, so the reflected signal is the same phase with the incident signal. The value of the reflection unit is 1 . From Fig 4 it shows that the value of the transport coefficient and the reflection coefficient is plus or minus only represents the phase of the reflected signal same or reversed from the 
incident signal, regardless of the transport direction of the signal[8].

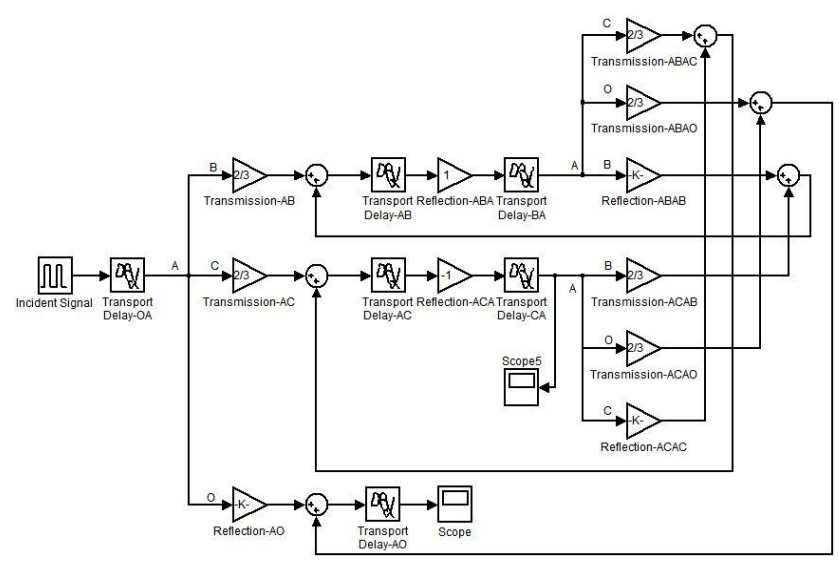

Fig. 4 The simulation model of the branched wiring networks

\section{The Simulation Model of the Branched Wiring Networks}

According to simulation model above, simulating the 2 branched wiring networks and 2 branched with 3 branches wiring networks simulation model in MATLAB/Simulink.

Using the amplitude of $1 \mathrm{v}$, a frequency of $1 \mathrm{MHz}, 50 \%$ duty ratio square wave signal as a high-frequency incident signal. And the wires of wiring networks are made of coaxial wires with the same characteristic impedance:
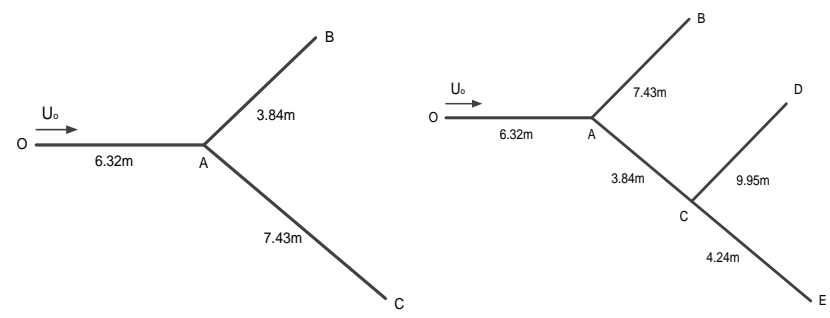

Fig. 5 Illustration of the branched wiring networks

In the simulation, the parameter values of the transport delay units and the reflection/transmission unit are set up by the various properties of the branched wiring networks: the length of the branch wires, the characteristic impedance of the wires and the transport speed of the signal in the wires[9]. Then run the simulation model to receive the reflected signal waveforms in Fig 6 and Fig 7:

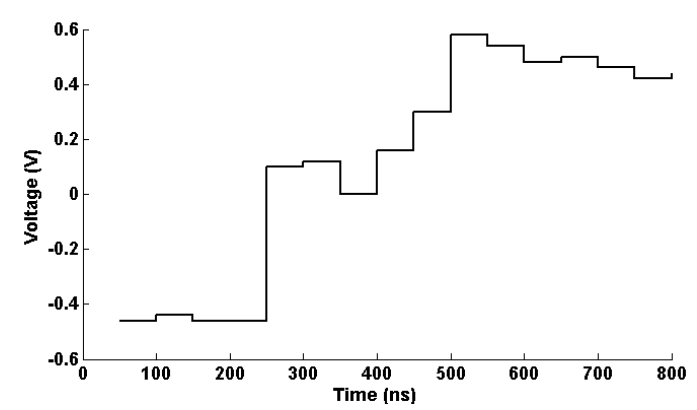

Fig. 6 The simulated reflected signal of 2 branched wiring networks

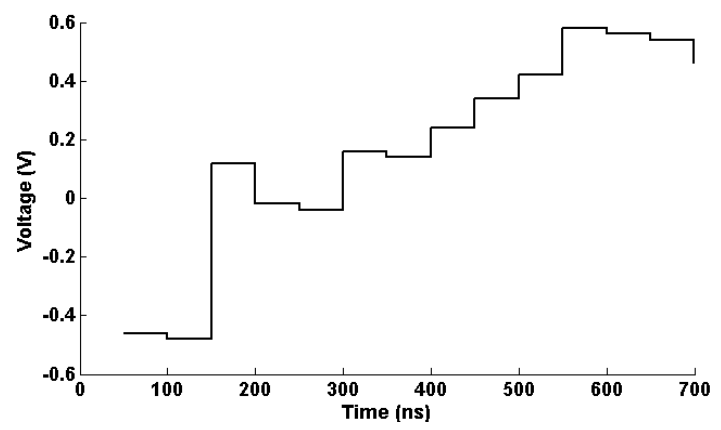

Fig. 7 The simulated reflected signal of 2 branched with 3 branches wiring networks

To measure the real reflected signal of the branched wiring networks. The Reflectometry detection system is composed of the PXI bus controller, the signal generate module, data acquisition module, the computer, the monitor and the T-joints, etc as shown in Fig 8:

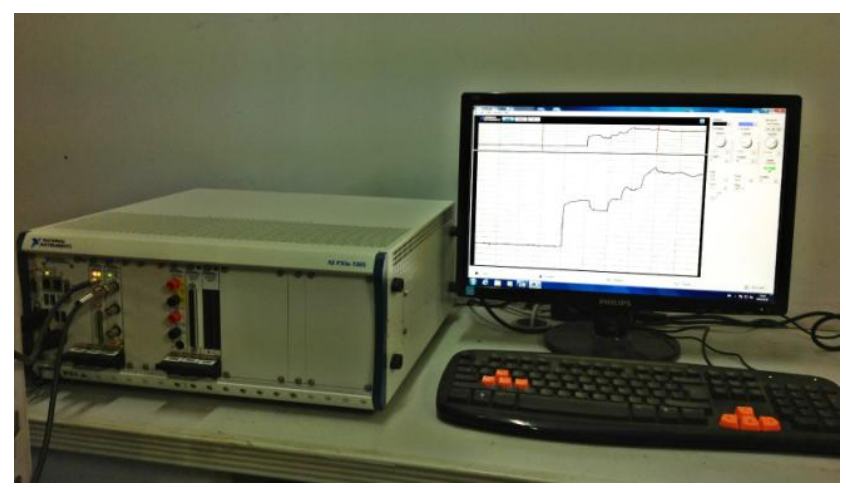

Fig. 8 Reflectometry detection system

Signal generate module generates the incident signal and the data acquisition module collects the reflected signal. The result is shown in Fig 9 and 10.

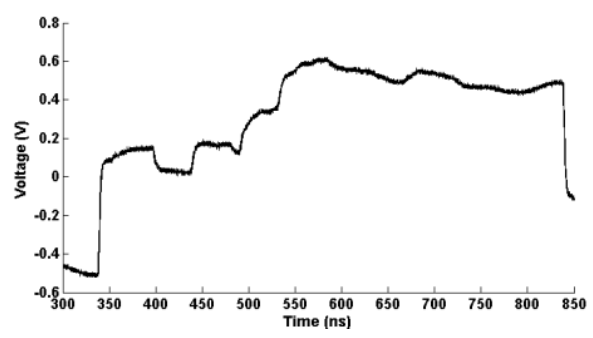

Fig. 9 The reflected signal of 2 branched wiring networks

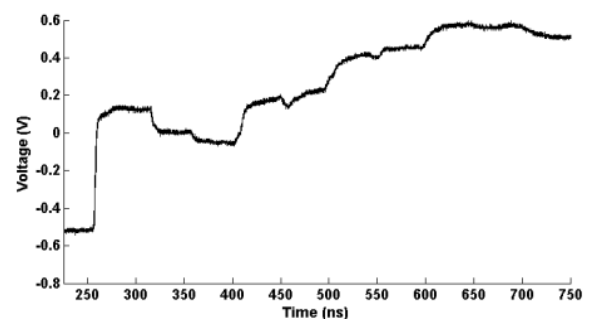

Fig. 10 The reflected signal of 2 branched with 3 branches wiring networks 
After a variety of different branched wiring networks simulation and measurement, run the simulated and measured reflected signals waveform matching operation to verify the accuracy of the simulation method.

The waveform matching algorithm uses the maximum correlation difference method(MCD)[10-11]. The specific algorithm is given by:

$$
\begin{aligned}
& \delta=\frac{\operatorname{Max}\left|\sum_{k=0}^{N-1} U_{k} U_{k+n}-\sum_{k=0}^{N-1} U_{k} X_{k+n}\right|}{\sum_{k=0}^{N-1} U_{k}^{2}} \\
& n=0,1,2, \cdots \cdot, N-1 \\
& \gamma=1-\delta
\end{aligned}
$$

In equation (4), $\delta$ and $\gamma$, respectively are the difference coefficient and similarity coefficient. $U$ is the simulation waveform data, $\mathrm{X}$ is the measured waveform data.

The result of the waveform matching is as shown in Table 1:

Table 1. Waveform matching result of the branched wiring networks

\begin{tabular}{|l|l|c|}
\hline $\begin{array}{l}\text { The branched wiring } \\
\text { networks }\end{array}$ & Type of fault & Similarity coefficient $\gamma$ \\
\hline B open-circuit & \\
C open-circuit & $94.24 \%$ \\
\hline & $\begin{array}{l}\text { B short-circuit } \\
\text { C open-circuit }\end{array}$ & \\
\hline & $\begin{array}{l}\text { B short-circuit } \\
\text { C short-circuit }\end{array}$ & \\
\hline & $\begin{array}{l}\text { B open-circuit } \\
\text { D open-circuit } \\
\text { E open-circuit }\end{array}$ & $93.57 \%$ \\
\hline
\end{tabular}

From the above diagram, the results shows that the similarity coefficients are within the threshold range regardless of the branched wiring networks or the types of fault. And as the complexity of the branched wiring networks, the value of similarity coefficients decrease. The conclusion can be drawn that this method to simulate the branched wiring networks faults is feasible. It can accurately simulate the propagation of the signal in branched wiring networks and the reflected signal of the faults in the branched wiring networks.

\section{Conclusion}

This paper has described a method to modeling and simulate the fault of aviation branched wiring networks. According to the characteristics of aviation branched wire network faults, establishing a mathematical model of the branched wire network faults based on the reflectometry. It can be used in analyzing the propagation of the signal in branched wiring networks, the characteristic of the reflected signal and the conditions of the faults.

Modeling and simulation results show that this method is accurate and effective. The simulation method provides a simulation model and theoretical basis for the future research of aviation branched wiring networks fault locating research and analysis.

\section{Acknowlegment}

This work is supported by Joint Funds of the National Natural Science Foundation of China and Civil Aviation Administration of China Key Project (Grant No. \#U1233201); The Innovation Fund project of National Commercial Aircraft Manufacturing Engineering Technology Research Center(Grant No. \#SAMC13-JS-15-017); Key Project of Tianjin Key Technology R\&D Program (Grant No. \#11ZCKFGX04000); The Fundamental Research Funds for The Central Universities (Grant No. \#ZXH2012B002 \& No. \#3122013P005).

\section{References}

[1] Brown M, F Gau. Wire Integrity Programs and Aging Aircraft Sustainment. The Joint 5th NASA/FAA/Dod conference on aging aircraft. Orlando. 2001.

[2] Smith P, Magelby A, etc. Non-destructive Fault Location on Aging Aircraft Wiring Networks. IEEE Antennas and Propagation International symposium. Columbus. 2003

[3] Feng K, LiWen W. Design and Implementation of Airplane-cable Fault Locator Used by Civil Aviation. Journal of Civil Aviation University of China. vol. 20, no.6, pp. 6-10. 2002.

[4] Boets P, Biesen LV. The Modeling Aspect of Transmission Line Networks. IEEE Instrumentation and Measurement Technology Conference. New York. pp.137-141. 1992.

[5] Furse C, Lo C. Wire Network Mapping Method and Apparatus Using Impulse Responses. US 7282922B2(Patent). 2007.

[6] JunFa M, ZhengFan L. Analysis of the Time Response of Multiconductor Transmission Lines with Frequency-dependent Losses by the Method of Convolution-characteristics. Microwave Theory and Techniques. vol. 40, no. 4, pp. 637-644.1992.

[7] JunMin Z, Juan W, etc. Based on Time Domain Reflectometry aviation wire insulation fault detection and analysis. Acta Aeronautica et Astronautica Sinica. vol.30, no.4, pp. 706-712.2009.

[8] Dingyu X, Yangquan C. System Simulation Technology and Application Based on MATLAB / Simulink. Tsinghua University Press. pp. 217-219. 2002.

[9] Lo C, Furse C. Modeling and Simulation of Branched Wiring Networks. Electromagnetic Compatibility, 17th International Zurich Symposium on. IEEE, 2006: 53-56.

[10] Escalona OJ, Mitchell RH. Frequency Bandwidth Limitations in the Signal-averaged ECG by the Maximum Coherence Matching Technique. Medical and Biological Engineering and Computing. vol. 31, no. 1, pp. 137-146.1993.

[11] Craelius W, Restivo M, etc. Signal Processing Options for Detecting Conduction Abnormalities in Ischemic Ventricles. Journal of electrocardiology. vol. 20(sl), pp. 119-124. 1993. 